\title{
La legítima defensa en favor de animales: análisis caso ecuatoriano.
}

\author{
The self-defense in favor of animals: \\ analysis of the ecuadorian case
}

\section{Jorge Vázquez Rodas*}

Investigador Jurídico Independiente

\section{Infomación del Artículo}

Original - Ruptura, 2020

Artículo recibido / Received: 9 de septiembre, 2020

Artículo aceptado / Accepted: 15 de diciembre, 2020

\section{Citación}

Vázquez, J. (2020) La legítima defensa en favor de animales: análisis caso ecuatoriano. Revista Ruptura de la Asociación Escuela de Derecho PUCE. Edición 2020, p (457-476).

DOI: $10.26807 /$ rr.vi02.8

Resumen: La consideración de los animales como titulares de derechos subjetivos propios forma parte de la evolución del derecho, aunque no exista unanimidad de criterios en la dogmática jurídica, la tendencia actual que puede ser evidenciada por reformas legales y resoluciones judiciales, apuntan al reconocimiento de derechos de los animales como personas no humanas. El texto trata de resolver la problemática que existe en caso de defender a un animal de agresiones antijurídicas provocadas por el ser humano. Se ha llegado a la conclusión que los animales poseen un bien jurídico tutelado por el derecho penal que, en caso de ser atacado las personas

* En la actualidad se encuentra cursando el noveno ciclo de la carrera de Derecho en la Universidad del Azuay y preside el comité de ética del Club Rotaract Gualaceo. Correo electrónico: vazquezrodasjorge@gmail.com 
pueden defenderlo amparados en una causa de justificación. Para llegar a esta conclusión se han resuelto las siguientes interrogantes: $i^{L}$ os derechos de los animales son un bien jurídico tutelado por el ordenamiento jurídico?, ¿Se puede reconocer la legítima defensa en favor de animales?, ¿Hasta qué punto puede llegar la legítima defensa para ser utilizada como medio de protección a un animal?

Palabras clave: Bien jurídico, legítima defensa, estado de necesidad, derechos de los animales, ordenamiento jurídico

\begin{abstract}
The consideration of animals as holders of their own subjective rights is part of the evolution of law, although there is no unanimity of criteria in legal dogmatic, the current trend that can be evidenced by legal reforms and judicial decisions, point to the recognition of rights of animals as non-human persons. The text tries to solve the problems that exist in the case of defending an animal from unlawful attacks caused by humans. It has been concluded that animals have a legal asset protected by criminal law that, in case of being attacked, people can defend it under a justification cause. To reach this conclusion, the following questions have been resolved: Are animal rights a legal asset protected by the legal system? Can legitimate defense in favor of animals be recognized? To what extent can legitimate defense to be used as a means of protection to an animal?
\end{abstract}

Keywords: Protected legal goods, self-defense, state of necessity, animal rights, legal system

\title{
Introducción
}

En los últimos tiempos, los derechos de los animales han logrado una mayor aceptación en todas las sociedades, al punto de que es casi imposible negar la existencia de los derechos que tienen estos, incluso existe un reconocimiento constitucional de vital importancia por el elevado valor que tiene la naturaleza y todos los seres que la conforman, entre ellos los animales. 
Con lo establecido en la Carta Magna, de manera muy sencilla, se observa la primera interrogante que surge al hablar de la legítima defensa en favor de animales, esta es: ¿̇los animales son titulares de un bien jurídico tutelado por el derecho penal?, este tema trascendental será abordado posteriormente en el texto, la importancia de tenerlo en cuenta en este momento es comprender la evolución constitucional que se ha tenido en el país, para analizar los alcances que puede tener respecto a la problemática que está siendo tratada.

Desde esta perspectiva constitucional, se entiende que la norma suprema llega a condicionar todo el ordenamiento jurídico. Como plantea Guastini (2003) se trata de la constitucionalización del ordenamiento jurídico, entendido esto como una constitución extremadamente invasora que tiene la capacidad de condicionar tanto la legislación y la jurisprudencia, de manera que todos los preceptos recogidos en ella deberán ser aplicables en todas las áreas del derecho, para que exista una armonía entre constitución y legislación. Una vez comprendida la perspectiva constitucional, que viene a ser la llave para que se reconozca la posibilidad de la legítima defensa en favor de los animales y haya la posibilidad de aplicarla en los casos presentados más adelante, con las condicionantes que influirán en la forma de realizarlo en uno u otro caso.

Partiendo del planteamiento anterior, se debe tener en cuenta la doctrina jurídico-penal, que la han venido desarrollando múltiples tratadistas, en el tema de la identificación del bien jurídico protegido en el caso de los delitos de maltrato animal; aunque no existe una decisión unánime, la posición actual otorga a los animales un bien jurídico propio. Al ser la doctrina fuente del derecho y específicamente del derecho penal, esta debe ser tomada en cuenta para el análisis.

El artículo número 28 del Código Orgánico de la Función Judicial en su último inciso establece:

Los principios generales del derecho, así como la doctrina y la jurisprudencia, servirán para interpretar, integrar y delimitar el campo de aplicación del ordenamiento legal, así como también para suplir la ausencia o insuficiencia de las disposiciones que regulan una materia. (COFJ) 
De manera que, todos los aportes doctrinarios en este tema, forman parte de la solución a la problemática que se plantea en este texto, al igual que sentencias internacionales a favor de animales, que constituyen los cimientos para que en un futuro estos temas tengan un total reconocimiento en el ordenamiento jurídico.

Para comprender a la legítima defensa basta con un análisis de lo establecido en el Código Orgánico Integral Penal en su artículo número 33 que expresa:

Existe legítima defensa cuando la persona actúa en defensa de cualquier derecho, propio o ajeno, siempre y cuando concurran los siguientes requisitos:

1. Agresión actual e ilegítima.

2. Necesidad racional de la defensa.

3. Falta de provocación suficiente por parte de quien actúa en defensa del derecho. (COIP)

De este concepto se deben seleccionar puntos importantes para que pueda existir la legítima defensa, debe cumplirse a cabalidad todos los requisitos establecidos por la norma legal para que la defensa de la víctima de una agresión antijurídica sea válida y le exima de responsabilidad. Por ello se aborda a breves rasgos cada uno de ellos, primero, la agresión debe ser actual e ilegítima, con lo que se entiende que debe ser cometida en el momento que la víctima aplica los medios de defensa. En lo referente a la actualidad de la agresión Roxin (1997) establece lo siguiente: “(...) una agresión es actual cuando es inmediatamente inminente, o precisamente está teniendo lugar o todavía prosigue (...)" (p.618). Dentro de este mismo requisito, se manifiesta que la agresión debe ser ilegítima, con ello se refiere a que la agresión sea contraria al derecho, por tanto, no cabe legítima defensa cuando la agresión es realizada de forma legítima; por ejemplo, el caso de la policía cuando siguiendo el uso progresivo de la fuerza llega a realizar agresiones contra los infractores. 
El segundo requisito es la necesidad racional de defensa por ello se entiende que existe una agresión por la cual la víctima necesita aplicar un medio de defensa contra el agresor, este debe ser racional; es decir, proporcional al ataque que está sufriendo, ya que, no puede extralimitar la legítima defensa. El tercer y último requisito, es la falta de provocación suficiente por parte de quien actúa en defensa del derecho, acarrea conflictos porque puede recaer en diversas subjetividades. Empero, para entenderlo se puede establecer que la legítima defensa será negada al agredido cuando la provocación que este realizó al agresor produzca determinada reacción del agresor y esta se entienda como la reacción normal que hubiera tenido una persona por ese tipo de provocación. (Muñoz, 2010)

Complementando lo planteado en el tercer requisito, es menester comprenderlo desde el planteamiento de Jakobs (1997), quien lo explica de la siguiente manera:

Se considera provocación sólo el comportamiento que fundamenta la corresponsabilidad por el ataque. Por una parte, tal es el caso en un ataque antijurídico del provocador, que a su vez desencadena un contraataque excesivo como defensa necesaria (p.487)

Analizados y comprendidos los requisitos es necesario volver a tomar en cuenta el artículo número 33 del Código Orgánico Integral Penal ya mencionado, de aquí se debe rescatar otro punto importante para la comprensión del alcance de la legítima defensa, por lo que, se expresa que está dentro de esta causa de justificación quien "actúa en defensa de cualquier derecho, propio o ajeno" (COIP), lo nuevo aquí es actuar en defensa de un derecho ajeno. Por lo que una vez analizada la legítima defensa es importante conocer que esta puede darse sobre terceros, de modo que, si cumple los requisitos mencionados anteriormente una agresión contra una persona o incluso contra bienes propios, se puede actuar para defenderlos.

En el caso de la legítima defensa de terceros, que tiene vital importación para el tema tratado, la doctrina a llega a un criterio uniforme y sin contradicción, como lo trata Molina (2012): 
Superadas etapas históricas en las que se acentuaba el carácter personal de la defensa, ligado al instinto de conservación, hoy es doctrina pacífica la que sitúa en plano de igualdad la defensa de intereses propios y ajenos y atiende para la justificación a los datos objetivos de la situación de conflicto, examinados desde la perspectiva de la lesividad para bienes jurídicos (p.21).

Por lo establecido ut supra, se descarta la duda en el caso de un animal que pertenece a la persona que lo defenderá de una situación de maltrato, es decir, de la propiedad de este, se puede ejercer la legítima defensa sobre el derecho de propiedad. Pero la gran interrogante que surge aquí es sobre animales que no sean de la propiedad de quien actuara en legítima defensa, se podría hacer de forma valida.

Esta interrogante será respondida con el siguiente análisis:

\section{I. ¿Los derechos de los animales son un bien jurídico tutelado por el ordenamiento jurídico?}

Esta interrogante va a tener enfoque en los casos que se ejerza la legítima defensa en favor de animales que no sean propiedad de quien actúa en beneficio de estos. Caso contrario tiene una respuesta sencilla, amparada en la defensa de un derecho propio como en el caso de la legítima defensa de bienes. Los animales, aunque de forma criticada, tienen un tratamiento de bienes muebles semovientes en el Código Civil ecuatoriano, su artículo numero 585 norma lo siguiente:

Muebles son las que pueden transportarse de un lugar a otro, sea moviéndose por sí mismas, como los animales (que por eso se llaman semovientes), sea que sólo se muevan por una fuerza externa, como las cosas inanimadas. (Código Civil, 2019)

Algunos países, como Alemania, han modificado su legislación civil en estos casos, siendo un avance progresivo en el tema, ya que el Código Civil alemán en el parágrafo 90 dice expresamente: 
Los animales no son cosas. Serán tutelados por leyes especiales. Se les aplicaron los preceptos correspondientes a las cosas sólo en medida de que no se disponga lo contrario. (Código Civil Alemán, 2020).

No obstante, en lo referente al caso ecuatoriano, al no existir otro tipo de regulación, el conflicto citado ut supra, se resuelve en virtud de la legítima defensa de bienes.

En los casos en que se debate el bien jurídico existente en los delitos de maltrato animal, se desprenden dos posturas contrarias: la primera corriente encuentra como titular del bien jurídico del maltrato animal a la sociedad, pues, esta es el objeto material del delito. Esto se da en virtud a que se está vulnerando el sentimiento social del pueblo civilizado, esta postura trae un inconveniente en el caso de la legítima defensa en favor de animales, pues, sería necesario atribuirle al animal la capacidad de ser sujeto de derechos; lo que se respalda en la segunda postura, el titular del bien jurídico son los animales mismos, atribuyéndoles el mínimo derecho a no ser sometidos a tratos crueles o sufrimientos innecesarios (Espina, 2020)

Un conflicto que se genera, y que debe subsanarse antes del análisis, es si cabe o no la legítima defensa. Por ello, en esta parte se analizará si el bien jurídico está amparado por ella. Por lo tanto, si el bien jurídico defendido en este caso, es comunitario o individual. En virtud de ello, existen tratamientos diferentes según la doctrina jurídico-penal. Puesto que se habla de bienes jurídicos comunitarios como plantea Muñoz (2010), cuando estos son indispensables para el orden social, en el que los individuos tienen que vivir, por ejemplo: salud pública, seguridad del Estado y orden público. Se habla de bienes jurídicos individuales, cuando se protege bienes que afectan directamente a un individuo en particular, por ejemplo: delitos contra la vida, el honor, contra el patrimonio, entre otros.

Esta clasificación a simple vista parece intrascendente, sin embargo, en virtud de ella, podemos distinguir cuales son los bienes jurídicos sujetos de protección en uso de la legítima defensa. Como bien resalta Greco (2019), la importancia de determinar si en este caso se trata de defender un bien jurídico colectivo o individual. 
Ese bien jurídico ha de adjudicarse a un individuo, los bienes colectivos no son susceptibles de ser protegidos en legítima defensa. De ese modo, solo se podría actuar en legítima defensa de los animales si estos fueran sujetos de derecho, así fuera en un sentido mínimo (Greco, 2019, p.27).

Asimismo, Zaffaroni (2011) señala que:

El bien jurídico en el delito de maltrato de animales no es otro que el derecho del propio animal a no ser objeto de crueldad humana, para lo cual es menester reconocerle el carácter de sujeto de derechos (p.49).

Con ello, se procede al análisis sobre si los animales tienen un derecho individual, por más rudimentario que sea; siendo este el punto de partida para reconocer la legítima defensa en favor de estos. Gracias al neo-constitucionalismo Andino, al cual el ordenamiento jurídico ecuatoriano está sujeto, por la constitución de 2008, se puede afirmar de forma sencilla el reconocimiento de derechos de la naturaleza, por lo que en la siguiente parte del análisis se constata esto.

Realizando un análisis de lo establecido en el párrafo anterior, es necesario el reconocimiento de los animales como sujetos de derechos que poseen bienes jurídicos penalmente protegidos, de modo que, pueden ser considerados alguien, en concordancia con lo que plantea Mir (2006):

En realidad la letra de la ley no consiente más límite que el del que se trate de la persona o derechos de alguien. No cabe la defensa de bienes suprapersonales, como por ejemplo el orden público (p.441).

Para ello, se consideran tres normas de gran importancia para este tema incorporados en la constitución ecuatoriana. Estos preceptos son la base, para afirmar que los animales tienen derechos subjetivos propios y que estos deben ser protegidos por los seres humanos. Los preceptos tomados son los siguientes:

1. El artículo número 10 que en su segundo inciso reconoce lo siguiente: "La naturaleza será sujeto de aquellos derechos que le reconozca la Constitución.” (CRE, 2008) 
2. El artículo número 71 en el cual se establecen factores de vital importancia para este reconocimiento:

"Toda persona, comunidad, pueblo o nacionalidad podrá exigir a la autoridad pública el cumplimiento de los derechos de la naturaleza" (CRE, 2008).

"El Estado incentivará a las personas naturales y jurídicas, y a los colectivos, para que protejan la naturaleza" (CRE, 2008).

Otra consideración importante, que debe ser tomada en cuenta, para poder determinar que los animales gozan de bienes jurídicos tutelados por el derecho penal, es que dentro del Código Orgánico Integral Penal, en los artículos 249 hasta el artículo 250.4, se establecen las contravenciones por muerte, maltrato, abuso y abandono de animales.

En concordancia con los preceptos planteados ut supra, Zaffaroni (2011) establece:

En efecto: si consideramos que el derecho penal no es constitutivo, sino que es sancionador, o sea, que no crea bienes jurídicos, sino estos le vienen dados por el orden jurídico, tampoco tiene autonomía para decidir quién es su titular. Esto se verifica apartando el código penal de la cuestión y comprobando que casi todas las conductas que tipifica no sólo constituyen delito, sino que también son ilícitos a la luz de alguna o algunas de las otras ramas jurídicas y, sobre todo, que ninguno de los bienes jurídicos lesionados deja de serlo si prescindiésemos de las tipificaciones (p.62).

Con ello se establece que el derecho penal por sí solo no crea los bienes jurídicos, sino, estos existen en todo el ordenamiento jurídico, por lo que, no se podría dejar de considerar a un animal como sujeto de derechos en el ámbito penal.

De igual modo, la doctrina es fuente del derecho penal, por lo que, debe ser tomada en cuenta al momento de resolver un caso, así que, con los argumentos precitados; los animales en la legislación ecuatoriana son sujetos de derechos y por lo tanto cuando existe maltrato hacia ellos se está vulnerando su bien jurídico propio como se expresa ut supra. 
En fecha 18 de diciembre de 2014, en Argentina se interpuso el recurso de habeas corpus en protección a una reconocida orangutana, llamada Sandra, en el zoológico de Buenos Aires. El mismo que tuvo un resultado favorable ya que finalmente se reconoce a Sandra la orangutana como sujeto de derechos; motivo por el cual se le debe adecuar su hábitat para su óptimo desarrollo. (Sala II de la Cámara Federal de Casación Penal, 2015).

De forma similar a la sentencia citada ut supra, está el caso de Chucho el oso, que de igual forma, gano un recurso de hábeas corpus ante la Corte Suprema de Justicia, Sala de Casación Civil de Colombia, en donde se obliga judicialmente al zoológico de Barranquilla a liberar y remitir inmediatamente a Chucho a un área que cumpla con las condiciones necesarias para su óptimo desarrollo. La Corte desarrolla que los animales son sujetos de derecho en tanto legislativamente han sido reconocidos como seres sintientes. (Sala de casación civil y agraria, 2017).

Con estas resoluciones se observa a dónde apunta la evolución del derecho en el mundo y esto es debido al reconocimiento pleno de los animales como sujetos de derechos, dejando de lado teorías que progresivamente van quedando descartadas.

Conforme a lo tratado hasta este punto, se puede concluir que los animales tienen derechos subjetivos propios y a su vez están protegidos por el ordenamiento jurídico-penal; en efecto, son titulares de bienes jurídicos. Estos son bienes que tienen gran importancia para la sociedad y en virtud de ello se genera su protección. Afirmando esto, se da paso al siguiente tema de análisis.

\section{II. $\quad$ iSe puede reconocer la legítima defensa en favor de animales?}

Por lo tratado anteriormente, se confirma que los animales gozan de bienes jurídicos protegidos. En efecto, se puede establecer que existe legítima defensa en favor de animales, cuando estos bienes jurídicos tutelados son vulnerados por parte de los humanos. 
Como primer punto, en virtud del reconocimiento constitucional, se acepta que los animales tienen derechos, por consiguiente, estos seres con vida se constituyen como portadores de un derecho subjetivo, por ello, dentro del tipo penal que regula la legítima defensa, podrían estar tutelados. De modo que, al conocer la incapacidad que estos tienen para poder defender sus derechos, de ataques por parte de los humanos, se concede dentro de la legítima defensa de terceros, que una persona pueda defender un derecho ajeno, en este caso, el de los animales.

Greco (2019), en su texto Legítima defensa de animales, equipara a los animales con los nascituros y los comatosos, pues estos son sujetos de derechos, pero tienen una incapacidad, que les impide percibir tales derechos, es por eso que requieren de una persona, ya que ella tiene la capacidad de conocer los derechos que estos tienen y la posibilidad de ejercer una defensa para que no sean vulnerados. La incapacidad de percibir sus derechos por parte de los animales es sobre todo intelectual, por tanto, no podrían ejercer una defensa en los casos de quebrantamiento de sus derechos.

Como manifiesta Greco (2019):

El auxilio al animal lo es en un doble sentido: primero porque el humano (capaz de defenderse) actúa en lugar del animal (por regla general incapaz de defenderse); segundo porque un defensor actúa a favor de un agredido, que en este caso es un ser vivo distinto a la especie humana (p.31).

Para el análisis de esta interrogante, que es trascendental para este estudio, es necesario plantear dos supuestos, en los cuales se analizará si se puede reconocer la legítima defensa en favor de animales, pues por lo expresado anteriormente se afirma que los animales tienen un derecho subjetivo propio; en efecto, ¿qué sucedería en caso de existir una vulneración sobre estos derechos?, ¿̇los seres humanos podrían actuar en su defensa? 
Casos para el análisis:

a. El primer supuesto: un grupo de activistas, por la protección de los derechos de los animales, ingresa a una granja para corroborar que en ella los animales se encuentran sometidos a tratos crueles y constantes. El objetivo de ello, es recolectar evidencia para denunciarla ante la autoridad competente.

b. El segundo supuesto: una persona que transita por las calles escucha lloros de dolor de un perro, empieza a buscar de donde proviene, y es cuando observa a una persona ahorcando al animal en su terraza.

En los dos casos existe maltrato animal, aunque la forma en que es producido es diferente, por lo que, debe tener respuestas diferentes, para determinar cuando el actuar de las personas es justificado y cuando no.

Es importante tener en cuenta, que a los animales se les debe conceder por lo menos dos derechos mínimos, en todos los casos, y sobre estos son los que se tratarán en este artículo. Estos derechos como bien los establece Greco (2019) en su texto son: "el derecho a no ser matado sin una razón justa o defendible, así como el derecho a vivir libre de sufrimiento o dolores constantes" (p.30).

Por medio de una entrevista realizada al especialista en bienestar animal, Maldonado (2020), quien establece que la forma de preservación mínima de los derechos de los animales, en el caso de aquellos destinados a la alimentación humana, es que durante su crianza perciban más emociones positivas que negativas. Asimismo, que no existan acciones $u$ omisiones que les generen sufrimiento constante durante su desarrollo; para la relevancia jurídico penal se debe tener en cuenta este tipo de actos humanos.

\section{a. $\quad$ Primer caso:}

Con esta breve explicación, de lo que significa el maltrato animal, se puede establecer en virtud del primer supuesto que se plantea, 
que existe una vulneración de derechos de los animales. Estos derechos deben ser protegidos por parte de los seres humanos. Efectivamente, los animales son sujetos de derechos, pero por su incapacidad (en su mayor parte intelectual), necesitan del ser humano para que los proteja.

El primer caso se analiza de la siguiente forma:

- Existen indicios sobre maltrato animal en la granja, por lo que el grupo activista se infiltra (cometiendo violación a la propiedad privada, tipificado en el artículo 181 del Código Orgánico Integral Penal), su finalidad es la obtención de pruebas para realizar la denuncia ante la autoridad competente. Los activistas abandonan el lugar una vez obtenidas las pruebas y estas son entregadas ante la autoridad competente (en el caso ecuatoriano sería el Gobierno Autónomo Descentralizado, que tenga competencia en ese lugar), para que esta intervenga. (COIP)

En este punto surge una interrogante, sobre si en este caso, existe legítima defensa o un estado de necesidad justificante. Greco (2019) resuelve el asunto de la siguiente manera, pues, dentro de la legítima defensa, no es un elemento necesario que exista una desproporción entre los bienes jurídicos, para que exista validez del actuar, lo que, si sucede dentro del estado de necesidad justificante, pues, en este supuesto, la desproporción de bienes legales es un elemento fundamental para que proceda.

Como plantea Muñoz (2010) “(...) el estado de necesidad justificante, que surge de la colisión de dos bienes jurídicos de distinto valor, cuando se opta del sacrificio de un bien de menor valor para salvar el de mayor valor (...)" (p.105). Se reconoce que los animales tienen derechos y que estos están jurídicamente protegidos, pero cuando existe un conflicto en el que involucra bienes jurídicos, que tienen diferente valor, como en el caso del animal y el ser humano, la forma adecuada de tratarlo, es por medio de la legítima defensa y no del estado de necesidad justificante. 
En efecto, existe la posibilidad de actuar amparado en una causa de justificación, en este caso la legítima defensa, pues el estado de necesidad justificante involucra que el valor de los bienes jurídicos sea diferente, y el que va a ser resguardado tiene que tener un nivel superior al que va a ser sacrificado; en caso de enfrentamiento humano contra animal, sería imposible aplicarlo, por la diferencia de valor de los bienes jurídicos protegidos, siendo la legítima defensa la mejor herramienta para esta situación.

\section{b. Segundo caso:}

- Una persona transita por la ciudad, cuando escucha llantos de dolor de un perro, la persona comienza a buscar de dónde provienen, logra divisar en una terraza a una persona ahorcando al animal, comienza a gritarle que se detenga y la persona no hace caso alguno. El ciudadano llama al 911 para denunciar esto y le ponen en espera, el decide entrar a esta casa al ver la puerta abierta (cometiendo violación a propiedad privada, tipificado en el artículo 181 del Código Orgánico Integral Penal), llega a la terraza y al intentar soltar al animal se produce un enfrentamiento entre las personas, la persona que llegó a evitar el maltrato del animal lesiona gravemente al agresor (cometiendo delito de lesiones, tipificado en el artículo 152 del Código Orgánico Integral Penal), y así logra soltar al perro y salvarlo. (COIP)

En este caso, se puede identificar con mayor facilidad que la persona actuó en legítima defensa, para salvar al animal, inclusive pidió al agresor que deje de hacerlo y al no acatar su petición, la persona intervino en defensa del animal.

La posibilidad de actuar amparado en la legítima defensa cumple una función de prevención general, en estos casos cuando se cumplen los requisitos que establece la ley, se puede actuar precautelando la vigencia de los derechos, evitando el ataque que puede darse en caso de omitir la defensa, concordante con lo planteado por Roxin (1997) que manifiesta: 
(...) al permitir toda defensa necesaria para la protección del particular, persigue simultáneamente un fin de prevención general; pues considera deseable que el orden legal se afirme frente a agresiones a bienes jurídicos individuales aunque no estén presentes los órganos estatales que estarían en condiciones de realizar la defensa (...) (p. 608).

Para explicar por qué en esta situación la acción de la persona se justifica rápidamente por medio de la legítima defensa, es necesario que se tome las palabras de Greco (2019), pues en este caso existen puntos determinantes, que influyen en que se lo pueda hacer de esta forma. "Para que se dé la legítima defensa en su forma característica deben reducirse todos los medios defensivos necesarios para acabar con la agresión de manera inmediata" (p.43).

En el caso planteado, al no haber respuesta por parte de la línea de emergencia, quedo reducido todo a la necesidad de su actuar para poder salvar al animal. Caso contrario a lo ocurrido en el primer ejemplo, pues los activistas necesitaban esas pruebas para poder denunciarla ante la autoridad competente, por lo tanto, no había forma de que una conducta como llevarse a los animales en ese momento fuera justificada, pues los casos son diferentes y la forma en que se produce la violencia también. En efecto, en el primer caso se puede obtener las pruebas para denunciarlo ante la autoridad competente y que esta sea la que intervenga, sin embargo, en el segundo caso esto cambia, pues, con la espera de la autoridad hubiera sido imposible proteger el derecho a la vida del animal.

Un punto importante que debe ser tomado en cuenta, en los casos en que se produzca enfrentamientos entre agresor y la persona que actúa en defensa de un animal, es la posibilidad que tiene el agresor de detener el ataque, ya que, siguiendo el ordenamiento jurídico estaría obligado a hacerlo, por lo tanto, el agresor al detener su conducta antijurídica, producirá que la defensa realizada por la otra parte se detenga.

Con lo dicho, se descarta totalmente cualquier intento de realizar justicia por mano propia, ya que, para aplicar la legítima defensa, 
existen elementos o requisitos que deben cumplirse a cabalidad, para que el actuar de la persona se encuentre justificado.

Por lo tratado hasta este punto, se puede concluir, que los animales son titulares de derechos subjetivos, pero por su incapacidad se vuelve totalmente complicado que ellos puedan percibirlos sin la ayuda de los humanos, por ello, y en reconocimiento de la legítima defensa de terceros, las personas pueden actuar en defensa de estos y estarán acogidos por una causa de justificación. Sin embargo, esto no es tan simple, porque las variantes podrían afectar cada caso en concreto, esto se analiza en la siguiente parte del texto.

\section{III. ¿ ¿Hasta qué punto puede llegar la legítima defensa para ser utilizada como medio de protección a un animal?}

Esta es una de las cuestiones más complejas dentro de la legítima defensa en favor de animales. Una vez corroborado que esta existe y que se puede ejercer, llega la interrogante ¿̇hasta qué punto puede practicársela?, es decir, la persona que actúa defendiendo legítimamente a un animal, dependiendo del caso concreto, ¿̇e podría actuar lesionando leve o gravemente al agresor?, o incluso en situaciones más extremas ¿se podría llegar al punto de atentar contra la vida del agresor por salvar a un animal?

Esta parte del análisis consiste en dos cuestiones, pues en el primer caso expuesto de la granja, existen lesiones crónicas y estables a los animales, es decir, el actuar inmediato de las personas, no sería trascendental para el caso y podría traer mayores conflictos que beneficios, por lo que, solamente se podría: invadir, documentar y salir. Pues caso contrario se podría incurrir incluso en lo que se llama justicia por mano propia, lo que es un absurdo total. (Greco, 2019)

En el segundo caso, que se analiza por la forma en que se produce, se requiere medios de auxilio inmediato, por lo tanto, demanda la actuación urgente de la persona en defensa del animal. 
No obstante, siempre las acciones de los defensores estarán limitadas, pues, no se puede ocasionar la muerte de la persona de ninguna manera, ya que según Greco (2019):

En este punto reconozco que la orientación antropocéntrica (los defensores de los derechos de los animales dirían: especista) del ordenamiento jurídico puede reflejarse en una limitación a la legítima defensa en la capa más externa. La defensa de un animal nunca podría justificar la muerte de una persona. En estos casos el ordenamiento jurídico asumiría una injusticia para no lanzar el falso mensaje de que animales y humanos tienen, de alguna manera, el mismo rango. Por supuesto cabría objetar que por qué se introduce la limitación aquí y no ya en los casos anteriores. No tengo una réplica contundente, sino solo la advertencia de que un ordenamiento jurídico solo debería ejercer su "Derecho a ser injusto" en los casos más extremos (p.41).

Lo planteado en esta parte del texto, deja una gran interrogante, que puede ser respondida por la diferencia que existe en el valor de los bienes jurídicos, pues, por la alta jerarquía que tiene la vida humana dentro del ordenamiento legal, no sería fácilmente aceptado sacrificarla por otro derecho. En efecto, el sistema jurídico tiene la facultad de ser injusto con el animal en este caso, debido al alto impacto social que produciría la muerte de una persona por salvar una forma de vida no humana.

Una forma clara de comprender que los bienes jurídicos no son equivalentes, es con lo expresado por Ávila Santamaría (2020), cuando respondió una pregunta sobre la legítima defensa de animales, y manifestó que, esta institución es una forma de saber los límites entre la especie humana con la naturaleza, de igual forma se refirió a que, se puede comer a los animales por una especie de estado de necesidad justificante, por tanto, con lo que se analizó anteriormente en este texto, se conoce que, en el estado de necesidad, existe una falta de equivalencia de los bienes jurídicos. Por tanto, no se podría justificar la muerte de una persona por salvar la de un animal. 
En consecuencia, la legítima defensa de animales siempre estará limitada por la desproporción que existe entre los bienes jurídicos protegidos, en estos casos, se reconoce que los animales tienen derechos subjetivos propios y que están protegidos por el ordenamiento legal. En efecto, se puede incluso provocar lesiones graves a una persona para proteger estos derechos, no obstante, en ningún caso se podría exceder el límite, que es la vida del ser humano, la cual es calificada como el bien jurídico protegido con mayor significación social.

\section{Conclusiones}

Reconociendo que los animales poseen derechos subjetivos propios y que estos por más mínimos que sean en el caso concreto, no podrán ser inferiores, de ninguna manera a dos derechos básicos, que están presentes en todo momento de la vida animal: el derecho a no ser matado sin una razón justa o defendible, así como el derecho a vivir libre de sufrimiento o dolores constantes. Con el reconocimiento de estas prerrogativas por parte del ordenamiento jurídico, se llega a la conclusión de que las personas pueden actuar para defender los derechos de las formas de vida no humanas, en virtud de la legítima defensa de terceros, pues, los animales tienen una incapacidad para poder percibir las prerrogativas constitucionales y legales que ostentan. Por ello, necesitan que los seres humanos intervengan en su defensa. En el presente trabajo, se equipará el derecho que los animales poseen, con el derecho de los nascituros, como forma de ejemplificar su incapacidad de entenderlos, arroparlos y ejercerlos, en el caso de los animales su incapacidad en mayor medida es intelectual, por lo que no pueden defenderse de algunos maltratos ocasionados por la especie humana. En el texto se realiza un análisis que tiene como conclusión que los animales son sujetos de derechos propios, siendo esto así, poseen un bien jurídico propio, el cual debe ser tutelado por la legislación penal. De modo que siguiendo la línea de evolución que está tomando el derecho, gracias a sentencias como de Sandra la orangutana o de Chucho el oso, y el crecimiento de la doctrina que respalda a los animales como poseedores de un derecho subjetivo propio. Al formar parte de las fuentes del derecho, sería el punto de partida para su aplicación en el Ecuador. 
Por lo antedicho, se reconoce la legítima defensa en favor de los animales, pero esta defensa siempre será limitada, pues, para proteger los derechos de los animales, las personas pueden actuar inclusive ocasionando lesiones graves al agresor, pero nunca podrían exceder este punto, por el nivel que tiene el bien jurídico "vida" para la sociedad. De modo que no se le podría otorgar la misma amplitud que tiene la legítima defensa de personas a los animales, por lo que se concluye que la vida humana se convierte en el límite de la legítima defensa animal.

\section{Referencias Bibliográficas}

Ávila, R. (2020, 15 de julio). Utopía del Oprimido [Webinar]. Universidad del Azuay. https://www.facebook.com/uazuay/videos/2683619365210780

Código Civil. (2019). Registro oficial . Ecuador : Asamblea Nacional.

Código Civil Aleman . (2020). Alemania : Parlamento Federal Aleman.

Código Orgánico de la Función Judicial . (2019). Registro oficial . Ecuador: Asamblea Nacional .

Código Orgánico Integral Penal . (2020). Registro oficial. Ecuador: Asamblea Nacional.

Espina, N. (Octubre de 2020). Legítima defensa de animales no humanos. En J. Pérez (Presidencia), Cátedra Jorge Rosell. Conferencia llevada a cabo en Universitas Fundación, Venezuela

Jakobs, G. (1997). Derecho penal parte general fundamentos de la imputación. Madrid: Marcial Pons.

Greco, L. (2019). Legítima defensa de animales. Nuevo foro penal, 15(92), 23-50. doi:10.17230/nfp.15.92.1 
Guastini, R. (2003). La constitucionalización del ordenamiento jurídico: caso italiano. En M. Carbonell, Neoconstitucionalismo(s) (págs. 49-73). España : Trotta.

Maldonado, M. (3 de Agosto de 2020). El bienestar animal. (J. Vázquez, Entrevistador)

Mir, S. (2006). Derecho Penal Parte General. Barcelona: Reppertor.

Molina, F. (2012). La legítima defensa del derecho penal. Revista jurídica Universidad Autónoma de Madrid, 19-48.

Muñoz, F. (2010). Teoría General del Delito. Bogotá: Temis.

Roxin, C. (1997). Teoría General del Delito Tomo I. Madrid: Civitas.

Sala II de la Cámara Federal de Casación Penal. (21 de Octubre de 2015) Sentencia A2174-2015/0. Juez ponente: Elena Liberatori

Sala de casación civil y agraria. (26 de Julio de 2017) Sentencia AHC48062017. Juez ponente: Luís Tolosa

Zaffaroni, R. (2011). La pachamama y el humano. Argentina: Madres de plaza de mayo. 